\title{
INFLUENCE OF SIMULATED MICROGRAVITY ON MECHANICAL PROPERTIES IN THE HUMAN TRICEPS SURAE MUSCLE IN VIVO
}

\section{Effect of 120-days of bed pest with physical training on human muscle contractile properties and musculo-tendinous stiffness in young women}

\author{
Yuri A. Koryak \\ SSC - Institute of Biomedical Problems RAS, Moscow, Russia \\ Address for corpespondence: \\ Yuri A. Koryak \\ SSC - Institute of Biomedical Problems RAS \\ 76-A Khoroshevskoye Shosse, 123007 Moscow, Russia \\ E-mail: yurikoryak@mail.ru
}

\begin{abstract}
Ahstract. Purpose. The aim of the study was to investigate the effects of a simulated microgravity on the mechanical properties of the human triceps surae (TS) and to assess the effectiveness of physical training (PT) in preventing detrimental effects. Methods. Eight women (aged 26-36 years) underwent 120-d of Bed rest (BR): four underwent Bed rest only (BR group) and four performed PT during this period (BRPT group). The training sessions were conducted for 60 min each day for 6 days a week for 14 weeks, and 30-40 min twice a day for 2 weeks under the experiment conditions. PT was performed over a 4-d cycle: $3-d$ of training and $1-d$ of rest. The maximal voluntary contraction (MVC), and isometric twitch contraction $\left(P_{t}\right)$, and electrically evoked tetanic tension at 150 impulses $\times \mathrm{s}^{-1}$ $\left(P_{0}\right)$, time-to-peak tension (TPT) of the twitch were determined. The difference between $P_{0}$ and MVC expressed as a percentage of $P_{0}$ and referred to a force deficiency $\left(P_{d}\right)$. The MTS was determined according to the electromechanical delay (EMD) value during the explosive voluntary contraction. Surface electrodes sensed electromyographic (EMG) activity in the soleus muscle. Electromechanical delay (EMD) was the time interval between the change in EMG and movement muscle force production. Results. In the BR group, $P_{t}, M V C$, and $P_{0}$ had decreased by $12,36,24 \%$, respectively, but $P_{d}$ had increased by $39 \%$. TPT of the twitch had increased by $14 \%$. The rate of increase of voluntary contractions reduced, but in the electrically evoked contraction no changes were observed. The EMD had increased by $27 \%$. In the BREx group, MVC, $P_{t}$, and $P_{0}$ decreased by $3 \%$, and $14 \%$, and $9 \%$, respectively. TPT had decreased by $4 \%$. The $P_{d}$ had decreased significantly by $10 \%$. The rate of rise increase of electrically evoked tetanic did not change significantly, but the rate of rise in isometric voluntary tension development was increased. The EMD had decreased by $12 \%$. Conclusion. Unloading decreased function and EMD muscle and, although the PT did attenuate these effects, they did not completely prevent them. It is suggested that the total loading volume (mainly its intensity) was not sufficient to completely prevent alterations in tendon mechanical properties
\end{abstract}

Key Worlls: bed rest, triceps surae muscle, training, electromechanical delay, musculo-tendinous stiffness, contractile properties 


\section{Introduction}

It is well known that the unloading of the musculoskeletal system by actual or simulated microgravity causes numerous changes in the musculoskeletal system, such as muscular atrophy and decreased contraction strength, both after relatively short-term (10-17 days) (Grigor'eva et al. 1987; Narici et al. 2003; Gopalakrishnan et al. 2010; Koryak 2008, 2011a, b) and long-term (>5 weeks) periods of unloading (LeBlanc et al. 2000; Kubo et al. 2000; Lambertz et al. 2001; Koryak 2007, 2011a, b). The deterioration of musculoskeletal function causes no direct health hazards and does not affect the capacity for work during short-term space missions. However, during long-term missions, serious problems can arise after returning back to Earth, unless the negative effects of microgravity are mitigated. Therefore, measures to prevent the complete adaptation of the human body to microgravity conditions are vital, as well as supporting the effective functioning of all body systems which have adapted to life in the terrestrial gravitational field through phylogenesis and ontogenesis.

Physical training (PT) has been proposed as a potential countermeasure to microgravity-induced effects on the size (mass) and function of skeletal muscles (Nicogossian 1982; Koryak 1998). High-resistance physical exercises are known to be effective in increasing the size/cross-sectional area or contractile force of muscles (Tesch 1991; Koryak 1993; Norrbrand et al. 2008; Fernandez-Gonzalo et al. 2014). Therefore, high-intensity resistance training may be successfully applied during unloading in order to counteract the deterioration of the contractile functions of the muscle.

Surface electromyogram data show the electrical activity of muscle and have been used in the analysis of human movement. The electromechanical delay (EMD) is traditionally defined as the time lag between the onset of muscle electrical activation and the onset of force production (Cavanagh and Komi 1979). Muscle force is registered only when the contractile elements of the muscle shorten, thus stretching the series elastic components (SEC), which participates in the transmission of force to tendons and joints (Hill 1938; Norman and Komi 1979; Cavanagh and Komi 1979; Hof and van den Berg 1981; Winter and Brookes 1991). The time required to stretch the SEC of the musculo-articular system is the prevailing EMD component and thus a measure of the series elastic stiffness. Changes in any of the previously listed events could potentially induce EMD alterations (Viitasalo and Komi 1981; Granata et al. 2000; Muraoka et al. 2004; Yavuz et al. 2010; Esposito et al. 2011; Cè et al. 2013; Lacourpaille et al. 2013).

The EMD changes can be attributed, first of all, to changes in muscle SEC stiffness. Muscle stiffness is described as the ratio between the force and length of stretching. A mechanically stiff muscle will transmit more force if the SEC stretches a small amount and, on the contrary, mechanically "gentle" (or "slack") tissue requires a significantly stronger muscle contraction in order to stretch the SEC and produce measurable force. "Slack" tissues require more time from the moment of activation to the start of force generation, i.e. their EMD is longer and it is shortened when muscle stiffness is increased before muscle tension (Norman and Komi 1979; Vos et al 1991; Zhou et al. 1995; Orizio et al. 1999, 2003). Therefore, the EMD may become a criterion for the differences in stiffness of the musculo-tendinous complex (MTC) under different conditions.

Unloading leads not only to the loss of muscle mass and muscle dysfunction (which seems to be clinically relevant), but also affects other functionally important structures of the musculoskeletal system, which undergo changes under the influence of microgravity (in particular, the tendon serially connected to the muscle). It is already known that the tendon not only provides structural connections between a muscle and a bone, but also plays the main role in the transmission of force produced by contractile muscle elements to the skeleton. Therefore, the 
tendon can change the length, and, therefore, the force of the contractile elements connected serially depends on the degree of variation of tensile strength (Zajac 1989; Reeves et al. 2004). The degree to which a tendon deforms in response to a muscle contraction depends on the mechanical properties of the tendon (Magnusson at al. 2001; Maganaris 2002; Maganaris and Paul 2002), since the tendon is not an inert structure and like a muscle it can change with the physical load level. The mechanical properties of the tendon may be adapted to changes in load level (Woo et al. 1980, 1982; Buchanan and Marsh 2001; Hansen et al. 2003; Reeves et al. 2003). For example, a higher load is associated with tendon hypertrophy (Woo et al 1980, 1982). Therefore, if unloading causes a decrease in musculotendinous stiffness (MTS), then it is vital to understand whether load (PT) can prevent the potentially harmful effect of unloading. The MTS is known to increase after strength training (Buchanan and Marsh 2001; Kubo et al. 2000, 2001; Grosset et al., 2009), and it may therefore be expected that the EMD would be shorter in those subjects who practiced PT in microgravity conditions, compared to those who were not subject to PT (Koryak 2012).

Firstly, the objective of this study is to evaluate the effect of PT on the contractile properties of the human triceps surae muscle (TS) in a group of young women in order to reduce the negative effects of mechanical unloading caused by long-term bed rest, and secondly, to assess the degree of EMD changes in the subjects of the study undergoing a strict bed rest regimen but also engaging in PT. We suppose that if the decreased MTC stiffness is caused by unloading, then PT during bed rest may prevent the detraining of skeletal muscles and reduce the negative effect of unloading.

\section{Materials and methods}

The study was approved by the Human Ethics Committee at the Institute of Biomedical Problems and had been performed in accordance with the principles of the 1975 Declaration of Helsinki on the use of human subjects in experiments.

\section{Participants}

Subject selection was based on a screening evaluation consisting of a detailed medical history, complete blood count, urinalysis, resting electrocardiogram, and a selection of blood chemistry analyses, which included the estimation of concentrations of fasting blood glucose, blood urea nitrogen, creatinine, lactic dehydrogenase, serum transaminase bilirubin, uric acid, and cholesterol, as well as an evaluation of their physical state using a bicycle ergometer stress-test. ${ }^{1}$ All subjects were recreationally active, but not involved in sporting activities at a competitive level. At the time of the study, no participant was taking any medication, and all were non-smokers. Eight women between 26 and $36 \mathrm{yr}$. of age gave their written, informed consent to participate in the study after they had been informed of all of the procedures and possible risks. The volunteers were allocated to two experimental groups: a group [ $\mathrm{n}=4$; age: 31.5 (SEM 1.7) yr.; body mass: 55.0 (SEM 1.8) kg; height: 162.3 (SEM 1.9) cm] that underwent Bed rest only (BR) and a group [ $\mathrm{n}=4$; age: 28.0 (SEM 1.1) yr.; body mass: 59.9 (SEM 2.3) kg; height: 162.3 (SEM 4.2) $\mathrm{cm}$ ] that underwent Bed rest while performing PT (BRPT). During preliminary visits to the laboratory, the subjects were informed of the objectives and methods of the study and were introduced to all the procedures for evaluating

\footnotetext{
${ }^{1}$ Continuous, gradually increasing work in the bicycle ergometer at a constant pedaling rate of $60 \mathrm{rpm}$ over 3 min with initial load of $50 \mathrm{~W}$ was specified; the load of subsequent "steps" was increased by $25 \mathrm{~W}$; achievement of submaximal heart rate was the criterion for work termination.
} 
voluntary muscle contractions and those induced by electricity. After that, each subject signed an Informed Consent Form for participating in the experiment. Each subject served as her own control.

\section{Bed rest}

Bed rest during 120-days in an anti-orthostatic position ( $5^{\circ} \mathrm{HDT}$ ) of the body was used as a model of the long-term hypokinesia/hypodynamia effect of spaceflight. The HDT position was chosen since various physiological alterations induced by actual spaceflight have been shown to be similar to those reported in ground-based studies using this model (Sandler and Vernikos 1986). During this 120-day experiment, the subjects were housed $24 \mathrm{~h}$ a day ${ }^{-1}$ in the Human Research Facility of the Health Ministry Institute of Biomedical Problems. During bed rest, the subjects remained in the HDT position continuously for all activities including excretory functions, showering, eating and PT.

\section{Exercise regimens}

Details of the training program and performance tests have been provided elsewhere (Eremin et al. 1970; Stepantsov et al. 1972). Briefly, subjects were accustomed to training using bilateral supine leg press and arm press. The PT consisted of three regimes: on the first day a force-velocity regime was carried out $(70 \%$ of the training was force-velocity exercise, and $15 \%$ a velocity and force requiring exercise); on the second day a velocity regime $(70 \%$ of the training was velocity required exercise, while $15 \%$ was force, and force-velocity requiring exercise), and on third day a force regime (70\% was force exercise, and $15 \%$ velocity and force-velocity requiring exercise).

The PT was scheduled over a 4-day cycle: 3 days of training and 1 day of rest. PT used during long-term spaceflights in the Russian space station "MIR" included a warm-up (walking on a treadmill for $5 \mathrm{~min}$ ), and low (2 $\mathrm{min}$ ), moderate (2 $\mathrm{min}$ ) and maximum (1 $\mathrm{min}$ ) intensity running. The training sessions were conducted for $60 \mathrm{~min}$ each day for 6 days a week for 14 weeks, and 30-40 min twice a day for 2 weeks under the experiment conditions. The recommended workload and intensities of exercises on treadmill varied from 3100 to $4400 \mathrm{~m}$, performed with an average rate of 117-135 m (Kozlovskaya and Grigoriev 2004). It is known that intensity of loads set up by the subject's level of work load, durations of high intensity intervals and subsequent pauses give a fairly correct notion about working abilities. In addition, taking into consideration the anatomical and physiological specificities of a woman's body, the total physical load was reduced to $70 \%$ of that usually exposed to by men, and when the expanders were used for muscle-strengthening exercises, the load was reduced by $25-30 \%$.

\section{Experimental protocol and measurements}

Experimental set-up. The subjects were carefully familiarized with the test procedures of voluntary force production during several warm-up contractions preceding the actual maximal contractions and were allowed to habituate to the electrical stimulation procedures during preliminary visits to the laboratory before definitive control measurements were taken. To ensure standardization of position and fixation of the limb during assessment, a special set-up, previously shown by Koryak (1985), was used (Figure 1). The dynamometer and recording system used to measure the forces produced by electrical and voluntary contractions of the TS have previously been described in detail (Koryak, 1985). In brief, the subject was seated comfortably on a special chair in a standard position (at a knee joint angle between the tibia and the sole of the foot of $90^{\circ}$ ). The position of the seat was adjusted to the individual and then firmly secured. A rigid leg fixation ensured isometric conditions for the muscle contraction. The dynamometer was a steel 
ring with a saddle-shaped block attached to fit the Achilles tendon. The resting pressure between the sensor and the tendon was constant for all the subjects and was set at $5 \mathrm{~kg}$. The contractile properties of the TS were tested twice: 10-8 days before the beginning of the Bed rest and after it ended. The test protocol was identical for both preBed rest and postBed rest tests.

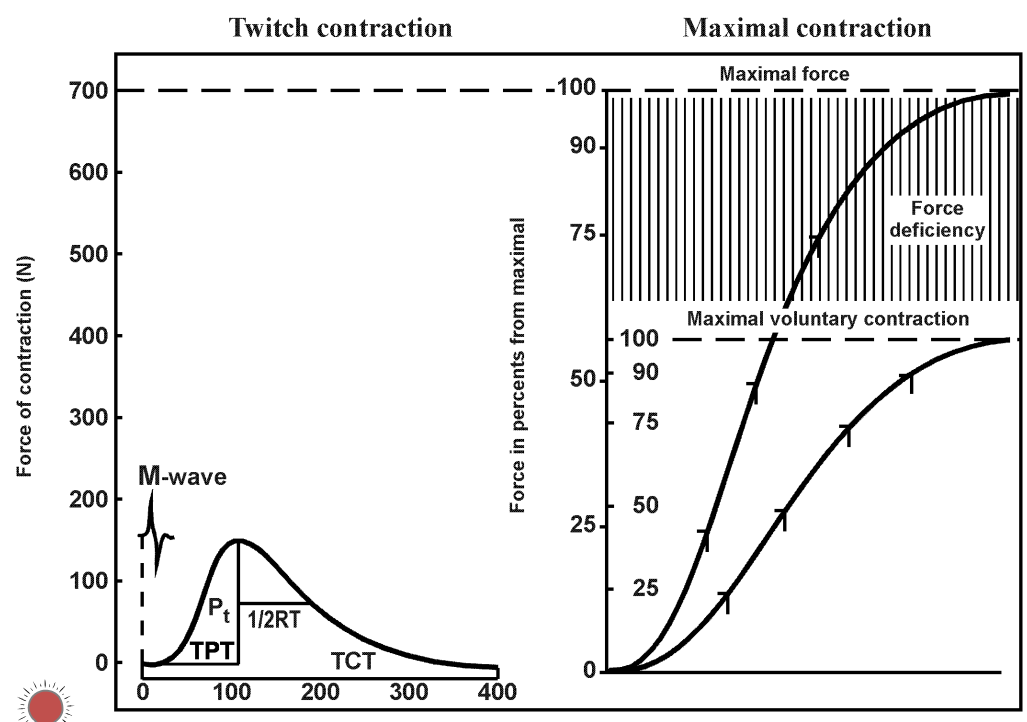

A

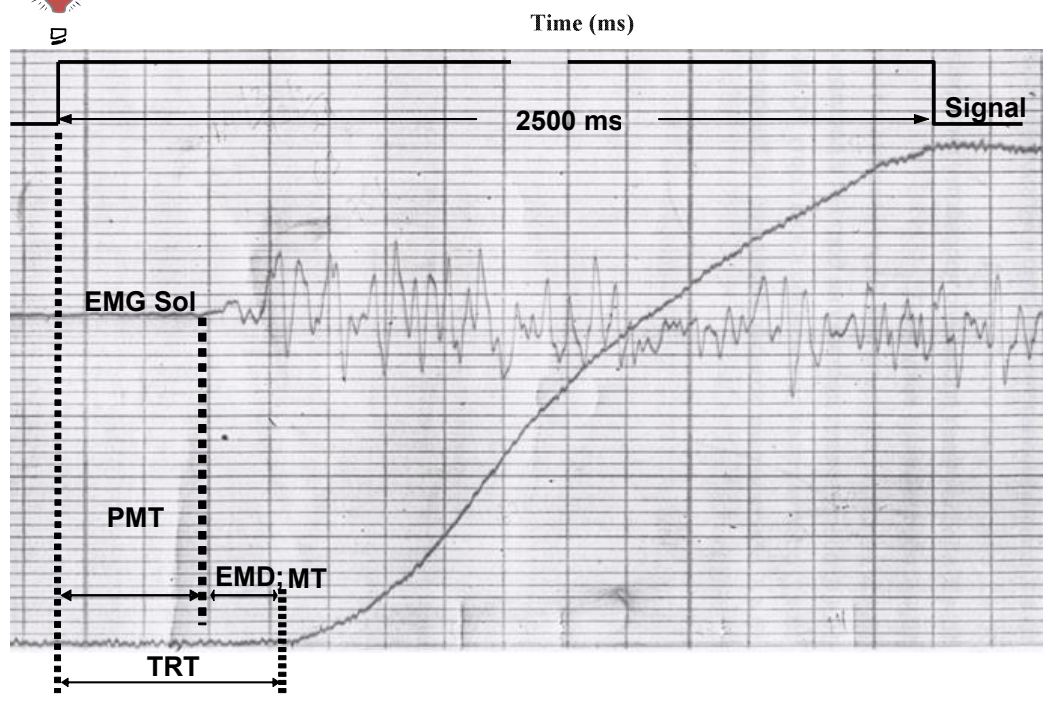

B

Figure 1. A. Examples of isometric twitch contraction curve (left) and electrically evoked tetanic tension and voluntary muscle tension development (right) showing how the parameters of the mechanical responses of muscle contraction were subsequently calculated. TCT, time-to-peak tension; 1/2 RT, half-relaxation time; TCT, total contraction time; $\mathrm{P}_{\mathrm{t}}$, twitch force. B. Schematic presentation of a sample showing total reaction time (TRT) with its premotor (PMT) and motor time (MT) or electromechanical delay (EMD) and electromyogram (EMG) of the soleus (Sol) muscle. 
Electrical stimulation. A single, square-wave, supramaximal transcutaneous electrical stimulus of $1 \mathrm{~ms}$ duration was delivered to the tibial nerve. Transcutaneous electrical stimuli were delivered to the tibial nerve using a high-voltage (maximal voltage $=400 \mathrm{~V}$ ), constant current stimulator (model "ESU-1", USSR). The cathode (active electrode) was a metal probe $(\varnothing 1 \mathrm{~cm})$ with the tip covered in a saline-soaked sponge, which was pressed over the tibial nerve in the popliteal fossa which is the place of the lowest resistance. The anode (passive electrode) was a $6 \times 4 \mathrm{~cm}$ rectangular self-adhesive electrode that was positioned between the tibial tuberosity and the patella. The large grounding electrode $(7.5 \mathrm{~cm} \times 6.5 \mathrm{~cm})$ was located in the proximal portion of the leg between the peak-up and stimulating electrodes.

Single stimuli were administered to the tibial nerve at a low current (amperage $=20 \mathrm{~mA}$ ) to determine the optimal probe location based on the visual inspection of the compound muscle action potential (M-wave) and $\mathrm{H}$-reflex of the soleus muscle that were monitored on an storage oscilloscope. A single stimulus was given every $30 \mathrm{~s}$. Once the location was determined and marked, the maximal M-wave was achieved with incremental ( $5 \mathrm{~mA})$ amperage increases until a plateau in the peak-to-peak M-wave was observed after three successive amperage increases. To assure a supramaximal stimulus, $120 \%$ of the stimulus that elicited the maximal M-wave was used during the evoked twitch procedures.

The isometric tetanic contractions of the TS were induced by electrical stimulation of the tibial nerve using supramaximal rectangular pulses with a frequency of 150 impulses $\times \mathrm{s}^{-1}$ (Koryak 1995). All the recordings were made in a room at constant temperature $(\sim 22 \pm 1 \mathrm{C})$.

Electromyography recording. Bipolar surface electrodes (standard $\mathrm{Ag} / \mathrm{AgCl}$ electrodes, $8 \mathrm{~mm}$ in diameter, spaced $25 \mathrm{~mm}$ centre-to-centre) were placed $6 \mathrm{~cm}$ below the insertion of the gastrocnemii on the Achilles tendon for the soleus. The large grounding electrode $(7.5 \mathrm{~cm} \times 6.5 \mathrm{~cm}$ in size) was located on the proximal portion of the leg between the upper recording electrode and the stimulating electrodes. The skin was rubbed with an abrasive paste and cleaned with alcohol to reduce the inter-electrode impedance to around $5 \mathrm{k} \Omega$. Electrode gel was used with all surface electrodes.

Procedure. Contractile properties of the TS estimated on mechanical parameters voluntary and electrical (involuntary) contractions. The experimental protocol consisted of four parts.

1. Maximal voluntary contraction (MVC) was estimated according to the tendogram of isometric voluntary contraction performed on the instruction condition to exert maximal contraction. From 2-3 maximal contractions were usually recorded from each subject until maximal force contractions was obtained. There was a 1-2 min rest between the sets. The MVC was determined as the highest value of voluntary force recorded during the entire contraction. The force was recorded on magnetic tape.

The subjects were also carefully instructed to respond to an auditory signal by exerting MVC as rapidly as possible, and to maintain it as long as the signal was audible ( 1.5-2.0 s). In the force-time curves, the times taken to increase the force to $25,50,75$, and $90 \%$ of MVC were calculated (Koryak 1985).

Involuntary (electrically evoked) isometric contraction (twitch contraction, double and tetanic) of the human TS caused electrical stimulated tibial nerve, using a neuromuscular stimulator.

The isometric twitch and tetanic contractions of the TS were induced by electrical stimulation of the tibial nerve using supramaximal rectangular pulses of $1 \mathrm{~ms}$ duration. Maximal isometric twitch force $\left(P_{t}\right)$ was estimated according to the tendogram of the TS isometric twitch response to a single electrical stimulus applied to the tibial nerve (Figure 1, A, left panel). The maximal force $\left(P_{0}\right)$ was estimated by the tendogram from the evoked contraction in 
response to an electrical tetanic stimulation of the nerve, innervating the TS, with a frequency of 150 impulses $\times \mathrm{s}^{-1}$ (Figure 1, A, right panel). The difference between $P_{0}$ and MVC expressed as a percentage of the $P_{0}$ value and referred to as force deficiency $\left(P_{d}\right)$ as has been calculated before (Koryak 1985).

After an appropriate rest of $4-5$ min the motor nerve was stimulated at various intervals. Supramaximal twin stimuli at 330, 250, 200, 100, 50, and 20 impulses $\times \mathrm{s}^{-1}$ were applied (Koryak, 1985). The maximal strength (amplitude) of the muscle contraction was determined and expressed as a percentage of the twitch contraction.

Tetanic index (TI) was expressed as the relation of $P_{0} / P_{t}$ (Close 1972; Koryak 1985).

2. The time from the moment of stimulation to peak twitch (TPT), the time from contraction peak to halfrelaxation (1/2 RT) were calculated by the tendogram of isometric twitch (Figure 1, A, left panel). The accuracy of measurement was $1 \mathrm{~ms}$.

3. The subjects were also carefully instructed to respond to an auditory signal by exerting MVC as rapidly as possible, and to maintain it as long as the signal was audible $(\sim 1.5-2.0 \mathrm{~s})$. In the force-time curves, by the time taken to increase the force to $25,50,75$, and $90 \%$ of MVC were calculated (Koryak 1985). Similarly, the rate of rise of evoked contraction in response to electric stimulation of the nerve with a frequency of 150 impulses $\times \mathrm{s}^{-1}$ was determined (Koryak 1985) (Figure 1, A, right panel). The accuracy of measurement was $1 \mathrm{~ms}$.

4. On a light signal the subject carried out plantar flexor under condition of «to contract as it is possible quickly and strongly» (Figure 1, bottom panel). Voluntary contraction in response to a visual stimulus (flash lamp) was adopted as a rapid ballistic movement. The signal to movement of «explosive» character was the visual diode - lamp ( $\varnothing 7 \mathrm{~mm}, 1 \mathrm{~W}$ ) - was placed at eye level $1 \mathrm{~m}$ in front of the subject. Lasted signals were $2.5 \mathrm{~s}$ and the pause between the signals was random ranging from 1.4 to $5.0 \mathrm{~s}$. The threshold for force was $5 \mathrm{~N}$.

A separate timer was used to record the time interval from the presentation of the light signal to movement. The special timer allowing synchrony with presentation of a light signal to the beginning of movement to record the development of mechanical answer of the human TS, was used.

From the tendogram total reaction time (TRT), defined as the time interval from the application of the light stimulus to movement, was estimated. TPT was divided into pre-motor (PMT), defined as the time interval from the application of the stimulus to the change in electrical activity of the soleus muscle, and motor time (MT or electromechanical delay - EMD), defined as the time interval from the change in electrical activity in the soleus muscle to movement (Weiss 1965). The force thresholds were also taken as relative values of $2 \%$ from the maximum isometric force level of each contraction.

Subjects were permitted three practice trials separated by $30 \mathrm{~s}$ and in most cases the mean of three readings was used to determine TRT, PMT and EMD.

\section{Statistical analysis}

Conventional statistical methods were used for the calculation of means and standard errors of the mean. Differences between baseline (background) values of the subject and those post-exposure (bed rest) were tested for significance by Student's paired t-test. Valued are given as mean \pm S.E.M. in the text. Significant differences between means were set at the $p<0.05$ level. The percentage changes for pre- and postBed rest were calculated. 


\section{Resullts}

There were significant baseline differences between the BR and BREx groups for any of the reported variables.

\section{BR group}

The mean changes in TS tension under HDT are shown in Figure 2 (top panel) and reveal a significant decrease. Isometric $P_{t}$ decreased by a mean of $11.5 \%$ [pre 105 (SEM 12.8) N compared to post 85.3 (SEM 5.9) N; $p<0.05$ ], MVC by a mean of $36.1 \%$ [pre 307.1 (SEM 21.6) $\mathrm{N}$ compared to post 196.2 (SEM 22.6) $\mathrm{N} ; \mathrm{p}<0.01$ ] $\mathrm{N}$ and $P_{0}$ by a mean of $24.4 \%$ [pre 503.3 (SEM 55.9) $\mathrm{N}$ compared to post 380.6 (SEM 28.4) $\mathrm{N} ; \mathrm{p}<0.01$ ]. The $\mathrm{P}_{\mathrm{d}}$ increased significantly by a mean of $39.8 \%$ [pre 37.6 (SEM 4.7) \% compared to post 48.8 (SEM 3.7 ) \%; $p<0.01$ ] after HDT (cf. Figure 2, lower panel).
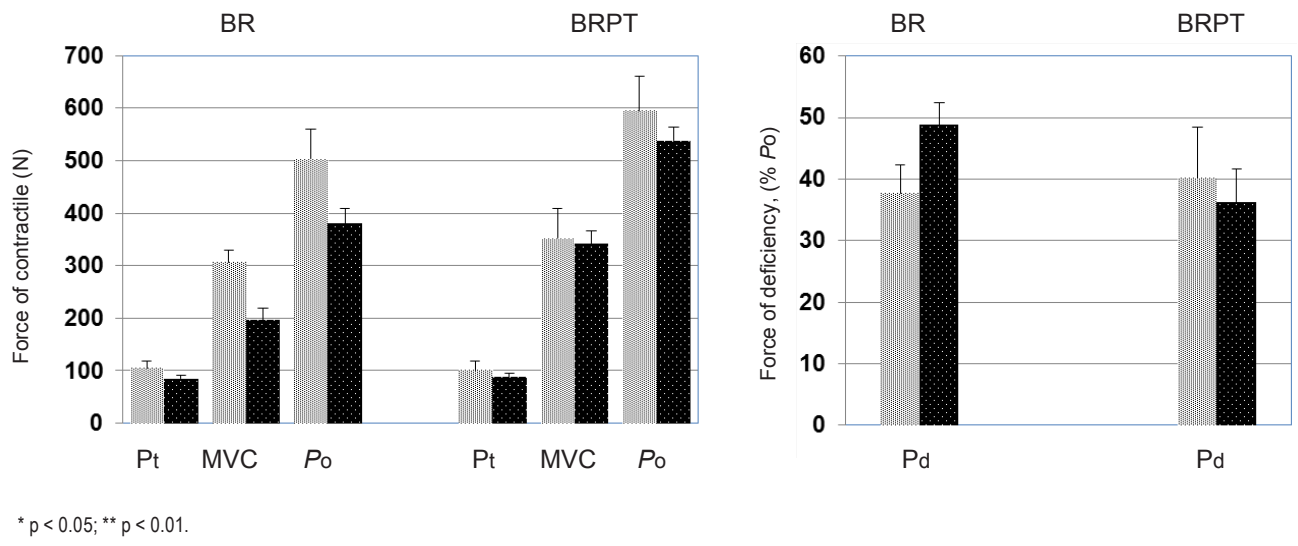

Figure 2. The effect of a 120-day $5^{\circ} \mathrm{HDT}$ for the BR and BRPT groups on maximal voluntary contraction (MVC), and evoked tetanic tension at $150 \mathrm{~Hz}\left(P_{0}\right)$, and at maximal twitch tension $\left(P_{t}\right)$ (top panel) and at force of deficiency (lower panel) of the triceps surae muscle. Values are means and S.E.M.

The change in mean time of isometric twitch contraction as the opposite value to contraction velocity for the TS after a 120-day HDT effect is given in Figure 3 (left panel). As is seen from the data analysis, exposure to HDT conditions was accompanied by a statistically significant decrease of the muscle contraction and increased relaxation velocity. Thus, TPT increased by a mean of $13.6 \%$ [pre 118 (SEM 5) ms compared to post 134 (SEM 5) ms], and 1/2 RT decreased by a mean of $19.2 \%$ [pre 123 (SEM 6) ms compared to post 102 (SEM 7) ms; $p<0.01$ ], respectively.

$\mathrm{TI}$ increased by a mean of $4.2 \%(p>0.05)$.

Mean changes in isometric force of the TS under paired stimulation of maximal intensity when twin stimuli were applied at 3, 4, 5, 10, 20, 50 ms separation are presented in Figure 4 (top panel). The greatest force of contraction was observed at intervals of 4-10 ms and decreases or increases of interval from this range was accompanied by considerable decreases $(p<0.05)$ with no change in the general pattern of muscle tension developed. At any given interpulse interval the relative increase in force of contraction after 120-day HDT effect was significantly less compared to the control value $(p<0.001)$. 




${ }^{*} p<0.05 ;{ }^{* *} p<0.01$

Figule 3. The effect of a 120-day $5^{\circ}$ HDT for the BR and BRPT groups on the isometric twitch time-to-peak tension (TPT), and halfrelaxation time (1/2 RT) of the triceps surae muscle. Values are means and S.E.M.

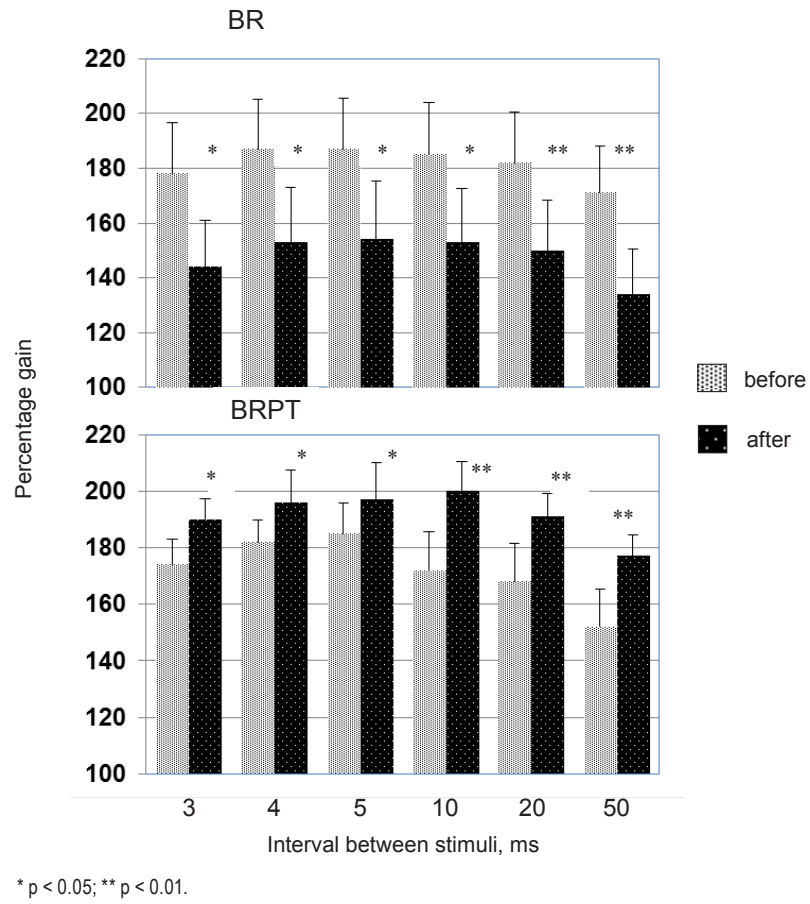

Figule 4. The effect of a 120-day $5^{\circ}$ HDT for the BR and BRPT groups on the maximal force contraction of triceps surae muscle in response to supramaximal twin stimuli at $3,4,5,10,20$, and $50 \mathrm{~ms}$. Values are means and S.E.M. 
Mean changes in the rate of development of isometric tension of the TS are given in Figure 5 (top panel). The analysis of the data gives evidence of a decrease $(p<0.01-0.001)$ in the rate of rise in isometric voluntary tension development of the TS (top panel). This may be seen as a decrease in the convexity of the force-time curve estimated according to a relative scale. However, in the assessment of the force-velocity muscle properties, no substantial changes were observed on the effect of 120-day HDT on isometric electrically evoked tetanic development (below panel).

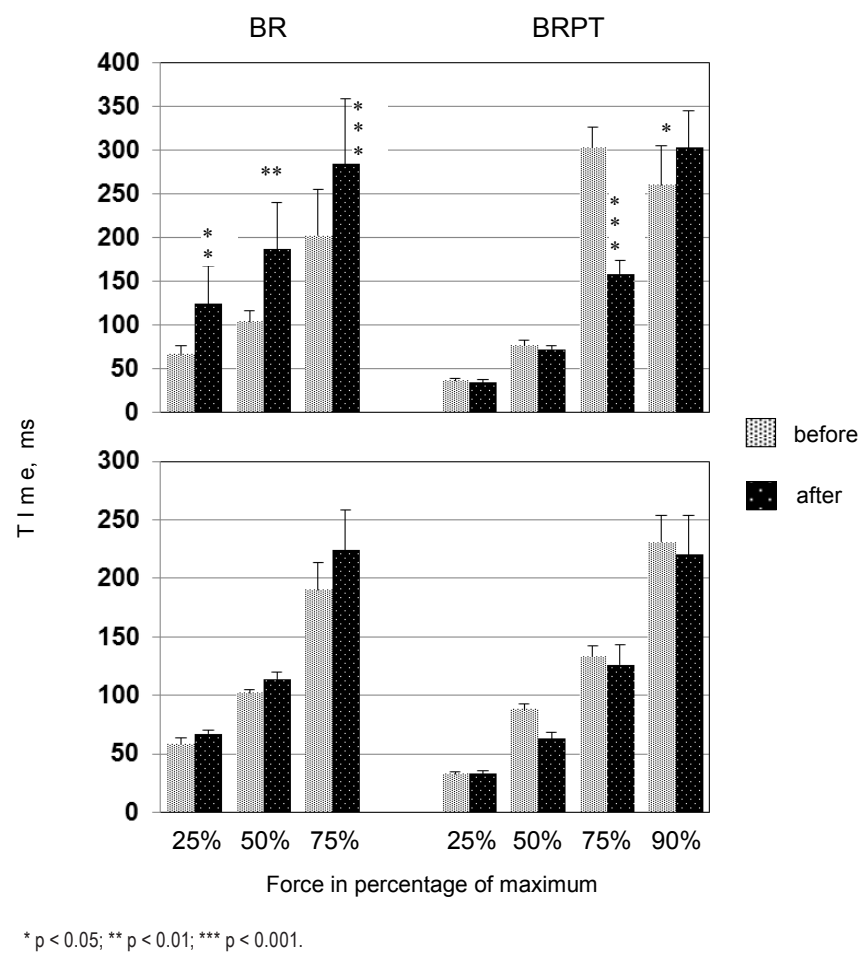

Figure 5 . The effect of a 120 -day $5^{\circ}$ HDT for the BR and BRPT groups on the maximal rates of electrically evoked tetanus (top panel) and of development of voluntary isometric force (below panel). Values are means and S.E.M.

The mean changes in TS EMD after a 120-day HDT effect is given in Figure 6 (left panel). The EMD increased significantly, by $27.4 \pm 1.3 \%$ after HDT (mean post HDT value $57.7 \pm 3.4$ ms compared to post mean pre HDT value $45.3 \pm 2.1 \mathrm{~ms} ; p<0.01$ ). The PMT decreased significantly by a mean of $21.4 \%$ [pre 167.4 (SEM 10.1) ms compared to post 131.6 (SEM 16.2) ms; $p<0.01$ ], and TRT decreased by a mean of 13.7\% [pre 157.3 (SEM 15.2) ms compared to post 135.8 (SEM 13.5) ms; $p<0.05)]$ after HDT. 


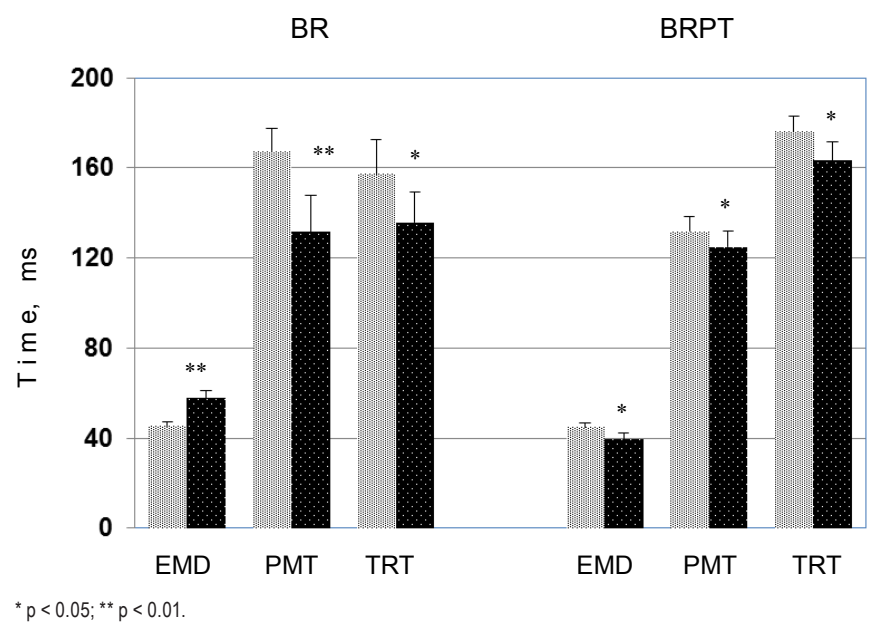

Figure 6. The effect of a 120-day $5^{\circ}$ HDT for the BR and BRPT groups on the total reaction time (TRT), premotor (PMT) and electromechanical delay (EMD). Values are means and S.E.M.

\section{BRPT group}

Mean changes in TS tension under the HDT effect are shown in Figure 2 (right panel), indicating a decrease in tension. Thus, isometric $P_{t}$ decreased by $13.6 \%$ [pre 101.0 (SEM 17.6) $N$ compared to post 87.3 (SEM 6.8) N; $p<0.05$ ], MVC by $3.1 \%$ [pre 352.2 (SEM 55.9) N compared to post 341.4 (SEM 25.5) N] and $P_{0}$ by $9.4 \%$ [pre 594.5 (SEM 66.7) $\mathrm{N}$ compared to post 538.6 (SEM 25.5) N]. The $P_{d}$ decreased by $10.0 \%$ [pre 40.2 (SEM 8.2)\% compared to post 36.2 (SEM 5.5)\%].

The change of mean time of isometric twitch contraction for the TS after the 120-day HDT effect with PT is given in Figure 3 (right panel). As it is seen the analysis of the data, exposure to HDT conditions was accompanied by a statistically significant increased muscle contraction and increased relaxation velocity. Thus, TPT decreased by $3.5 \%$ [pre 135.5 (SEM 11.7) ms compared to post 130.8 (SEM 6.0) ms], 1/2 RT decreased by 7.4\% [pre 101.5 (SEM 10.0) ms compared to post 94.0 (SEM 10.2) ms; $p<0.05$ ].

TI was reduced by $15.8 \%(p<0.05)$.

Mean changes in isometric force of the TS during paired stimulation of maximal intensity at interpulse intervals of $3,4,5,10,20$, and $50 \mathrm{~ms}$ can be seen in Figure 4 (low panel). As may be seen the greatest force of contraction was observed at intervals of 4-10 ms and decreases or increases outside this range was accompanied by considerable decreases $(p<0.05)$ but with similar trends of tension developed by the muscle. There were differences in the curve at identical interpulse intervals the relative increase in force of contraction after 120-day HDT with PT being significantly greater by comparison with the initial values $(p<0.001)$.

Changes in the rate of development of isometric tension in the TS are shown in Figure 5 (lower pane/). Analysis of the data provides evidence of an increase in the rate of rise of development of isometric voluntary tension in the TS $(p<0.05)$. This may be seen as an increase in convexity of the force-time curve estimated according to a relative 
scale. However, no substantial changes were observed in the electrically evoked isometric tetanic development (below panel).

The analysis of the maximum normalized ratio $\mathrm{d} P_{0} / \mathrm{dt}$ revealed a not significantly decrease by a mean of $8.4 \%$ [pre $0.83 \% / \mathrm{ms}$ compared to post $0.78 \% / \mathrm{ms}$ ]. The analysis of the development of electrically evoked contractions demonstrated not significantly differences throughout the whole period of the development of isometric tension, but at the same time, a minor (by a mean of $14.5 \%$ ) increase of the maximum $\mathrm{d} P_{0} / \mathrm{dt}$, compared to the initial one [pre $1.17 \% / \mathrm{ms}$ compared to post $1.34 \% / \mathrm{ms} ; \mathrm{p}<0.001]$.

The EMD was significantly less in response to the light signal (by a mean of $12.2 \%$ ) after 120 -day HDT with PT compared to the baseline value [pre 44.9 (SEM $2.0 \mathrm{~ms}$ ) compared to post 39.4 (SEM 3.1) ms; $p<0.05$ ] (Figure 6, right panel). After 120-day HDT, the PMT decreased by $5.3 \%$ (pre $131.8 \pm 6.4 \mathrm{~ms}$ compared to post $124.8 \pm 7.1 \mathrm{~ms}$; $p<0.05$ ) and the TRT decreased by a mean of 7.3\% [176.2 (SEM 6.7) ms compared to post 163.4 (SEM 8.3) ms; $p<0.05]$.

\section{Discussion}

The present study aimed to elucidate the effects of chronic unloading on the mechanical properties of human TS and to examine the potential preventive effects of PT performed during the period of unloading (of strict the 120-day HDT) on mechanical properties of the muscle. The TS was the muscle tested in the study, since it has been demonstrated that foot extensors were the muscles most affected by unloading, compared to other extensors of the lower limbs (Fitts et al 2000).

This study considers the program of physical exercises carried out by the study subjects during 120-day of strict HDT, while the International Space Station team members carry out a similar program over 6 months. We studied the advantages of this program in order to protect various contractile elements of the skeletal muscle. This study may be considered unique due to the duration of the unloading period (120-day) with the use of women as study subjects and the examination of the physical adaptation of the muscular system to longer periods of unloading.

The most significant finding of the present investigation was the absence of changes in the contractile properties, and contraction time, as well as in the rate of development of voluntary tension contraction of the TS after the 120-days of bed rest when an attempt was made to preserve muscle condition. For instance, the MVC after the 120-day HDT with PT decreased by $3 \%$, the TCT decreased by $4 \%$, and the rising curve of isometric voluntary contraction underwent fewer changes, which, in general, confirms the importance of PT in the preservation of muscle function, especially under conditions of mechanical unloading. However, the PT program applied, as well as the regimen of exercises carried out, did not completely prevent the negative effect of unloading on TS function.

The training programme applied in the present study had an effect on contractile properties in the TS. A significant decrease in MVC was observed after 120-day bed rest (Koryak 1995) and it was concluded, therefore, that the small decrease (3\%) in MVC after 120-day bed rest with PT reflected a neural adaptation. The decrease in $P_{d}$ (cf. Figure 3, right panel) would suggest an increase in central drive in the control of voluntary muscle by the motor nerve system. These findings taken together with the reported increase in motor unit synchronization (Milner-Brown et al. 1975) and motor unit activation (Komi et al. 1978; Moritani and DeVries 1979) would suggest that neural adaptation may also have contributed to the increase in voluntary strength that occurred with training under conditions of120-day bed rest. It was anticipated that the changes in $P_{t}$ and $P_{0}$ would correspond to these in voluntary 
force, namely, with increases in training (see Koryak 1993) and decrease with bed rest without countermeasures (Koryak 1995). In contrast, it was observed that there were nonsignificant changes in the twitch tension after 120-day HDT with PT (Koryak 1995). These unexpected experimental findings are not unique. Edgerton (1976) has found a nonsignificant $15 \%$ decrease in the gastrocnemius muscles twitch tension in the bushbaby. These changes are quite similar to those observed in our study.

PT counteracts the muscle mass loss related to inactivity and prevents changes in the composition of muscle fibers caused by disuse (MacDougall et al. 1980). This agrees with the previous suggestion of Alway et al. (1988) that contractile adaptation to training can occur independently of the changes in percentage fibre type. Since we were not able quantitatively to assess the intensity of the exercises performed and the device applied had certain limitations, this could have led to the study subjects performing a greater volume of exercises, but with less intensity. Data on the use of high resistance exercises with high intensity confirms their importance in the protection of muscle function from atrophy. Thus, balanced PT consisting of high resistance and intense exercises (2-3 days/week) and aerobic exercises ( 4 days/week) is more effective in the preservation of muscle function during prolonged bed rest (Trappe et al. 2007, 2008, 2009). Moreover, training with highly intensive resistance exercises lasting for $\sim 7 \mathrm{~min} /$ week during the bed rest period was more effective when compared to exercises lasting for more than $60 \mathrm{~min} / \mathrm{week}$ under actual zero gravity conditions (Trappe et al. 2009).

It was anticipated that the changes in $\mathrm{P}_{\mathrm{t}}$ and $P_{0}$ would correspond to these in voluntary force, namely, with increases in training (Koryak 1998) and decrease with Bed rest without countermeasures (Koryak 1998). In contrast, in the present study, $P_{0}$ even slightly decreased $(\sim 9 \%)$ after PT, which led to a tendency toward a decrease in the $P_{t} / P_{0}$ after the 120-day HDT with PT. Changes in the $P_{t} / P_{0}$ may reflect the degree of changes in the muscle tensile strength. The literature indicates that disuse induced an increase in muscle and joint stiffness and a decrease in the range of motion (Lambertz et al. 2003; Grosset et al. 2010). Decreased muscle tensile strength permits the body to transmit the tension developed by sarcomeres of the contractile tissue more effectively and thus the $P_{t} / P_{0}$ increases. On the contrary, increased muscle tensile strength during PT may result in a decreased $P_{t} / P_{0}$ (Less et al. 1977). We suppose that in our experiment PT during the HDT contributed to the increased muscle tensile strength to a certain extent. Training may lead to a decreased $P_{t}$ by causing an increase in the muscle tensile strength, which is described in this work.

The PT applied in the present study was performed every third day, which only allowed the subjects to carry out two sessions a week. Furthermore, taking into consideration the fact that physical training was performed twice a week by the end of the study but total physical load was reduced down to $70 \%$ of that usually applied to men (see the Methods), then it seems that the total volume and, especially, the intensity of exercises, was insufficient to support the mechanical properties of the TS tendon. Furthermore, PT significantly reduced the level of decrease in MTC stiffness when compared to unloading without training (Koryak 2012). It should be noted that the tendon is not an inert structure, and a skeletal muscle also changes with the level of physical load; in other words, the mechanical properties of the tendon may adapt to load changes.

During gravity loading $(0 \mathrm{G})$, the human TS tendon is exposed to very high rhythmic load due to constant muscle use and the transmission of muscle force related to heel activity (heel-and-toe movements), which require stabilization and movement of the body during movement (walking and running) (Fukunaga et al. 2001). Therefore, during unloading the full volume of exercises (load level, frequency and duration) should exceed the threshold level in order to prevent changes to the mechanical properties of the tendon completely. The walking load produced by 
the Achilles tendon is $\sim 210 \mathrm{~kg} / \mathrm{cm}^{2}$ (Finni. et al 1998), i.e. for a person weighing $70 \mathrm{~kg}$, the lifting and dropping of body weight in $1 \mathrm{~g}$ would lead to the development of tension in the Achilles tendon equal to $200 \mathrm{~kg} / \mathrm{cm}^{2}$. Therefore, if we suppose that ordinary walking is a stimulus sufficient to maintain the mechanical properties of the tendon under normal gravity conditions, then the threshold PT level required for the prevention of any deterioration during unloading should be more than or equal to the body weight. Hence, it may be supposed that although the load level applied in this study has been sufficient, the training regimen followed during unloading has been significantly lower than the load frequency during ordinary walking. Therefore, the required load frequency should obviously be higher in order to exceed the threshold volume. The latter is confirmed by a study when during 20 days of bed rest, PT was not only performed every day (16 out of 20 days), but also with load/weights approaching the subjects body weight (Akima et al. 2003). Moreover, the analysis of exercises used in the training process within the frames of this study revealed a lack of exercise for training the foot extensors. And if we take into consideration the fact that the total PT load was reduced (see the "Methods"), it may be the main (or even principal) cause of incomplete preservation of muscle function during unloading.

PT and disuse have often been associated with muscle hypertrophy and atrophy, respectively (MacDougall et al. 1980). In the present experiment no direct measurement of the TS mass muscle was made; however, the peakto-peak amplitude of the maximal $\mathrm{M}$-wave is an indirect measurement of the excitable muscle mass. The M-wave amplitude did not change significantly with either PT or HDT [10.4 (SEM 0.9) mV compared to post 11.0 (SEM 1.0) $\mathrm{mV}$ ], suggesting that little alteration in muscle mass occurred.

During many everyday activities, the ability to produce high muscle force per time unit is more important than the ability to generate high force. The rate of force development depends on many factors, in particular, on the duration of the excitation-contraction process, on the force-velocity properties of muscle fibers (even during isometric contraction due to tendon structure deformity) and SEC stiffness. Therefore, decreased MTC stiffness may be the reason for the decreased rate of development of TS contraction after unloading, since SEC stiffness in known to be an important factor determining the rate of muscle force development (Bojsen-Møller et al. 2005). In other words, as indicated in the Introduction, the time of SEC stretching by contractile elements determines the EMD value (Hill 1938). Therefore, changes in MTC (SEC) stiffness after hyperactivity/hypoactivity mainly explain changes in EMD. In addition, all the structures of the SEC, classically composed of an active part (located in myofibrils) and a passive part (mainly aponeurosis and tendon) (Zajac 1989), could contribute differently to EMD. Nordez et al. (2009) used the noninvasive methodology (a real-time ultrasonography) determine the relative contribution of the passive part of the SEC $(47.5 \pm 6.0 \%$ of EMD) and each of the two main structures of this component (aponeurosis and tendon, representing $20.3 \pm 10.7 \%$ and $27.6 \pm 11.4 \%$ of EMD, respectively).

This study demonstrated that the rate of isometric voluntary contraction development performed after the instruction to exert the fastest contraction had decreased after unloading, which confirms the theoretical relationship between the decreased muscle MTC stiffness and decreased rate of transmission of the contractile force and therefore the rate of contraction development, but to a significantly less extent when compared to similar conditions without PT (see Figure 4). This data was obtained for the first time and is indicative of the increase of MTC stiffness after PT under conditions of muscular system unloading. Our results in the present study indicate that PT during bed rest results in an increase in MTS (MTC), whereas a lack of training is associated with a decrease of this parameter (Koryak 2012). Therefore, PT decreases the EMD under muscular system unloading conditions. 
The changes in elastic properties due to PT have been well documented. Endurance training resulted in an increase in the SEC stiffness in the soleus muscle of rats, associated with an increase in type I fibres (Goubel and Marini 1987). Both jump and endurance training also appear to increase both collagen concentration (Kovanen et al. 1980; Ducomps et al. 2003) and muscle passive stiffness. The soleus rat muscles submitted to plyometric training had more fast twitch fibres and a lower SEC stiffness than controls (Watt et al. 1982; Pousson et al. 1991; Almeida-Silveira et al. 1994). Malisoux et al. (2006) reported that human subjects given 8 weeks of maximal effort stretch-shortening cycle exercise training tended to have an increase in the proportion of type II fibres in their vastus lateralis muscles. Kubo et al. (2000) reported that the MTS is greater in long distance runners than in untrained individuals.

The results obtained correlate well with data on the decreased EMD after isometric training under bed rest conditions (Kubo et al. 2001). The EMD changes during training are mainly attributed to changes in the tendon structure, but it should be noted that tendon stiffness is always increased during any physical activity, both during endurance (Buchanan and Marsh 2001) and isometric training (Kubo et al. 2000, 2001). Furthermore, EMD changes correlate very closely with muscle MTC and especially with the active SEC fraction during training (Pousson et al. 1991; Ducomps et al. 2003, Malisoux et al. 2006).

This study demonstrated that the EMD is sensitive enough and may serve as an indirect marker for measurements of muscle MTC stiffness in order to determine the chronic adaptation of the musculo-tendinous structure of the muscle to the mechanical unloading during PT under real or simulated microgravity conditions. The data obtained demonstrates that a 120-day of simulated microgravity led to decrease the TS mechanical properties and although the adverse effects were reduced thanks to PT, they were not completely prevented by the training program. This permits us to suppose that the volume of exercises and especially the intensity of exercises performed did not exceed the threshold level required for the complete prevention of changes in mechanical properties.

The mechanical muscle responses obtained and the list of physical exercises received from the study subjects, as well as from members of long-term (6-month) space missions confirm the importance of PT in preserving muscle function and the capacity to work during long-term stays under microgravity conditions. Within the framework of this study, the PT program contained mainly low intensity exercises. The inclusion of exercises with higher load and higher intensity into the training process would contribute to a more effective exercise program for the training of skeletal muscles and it would reduce the total training time under zero gravity conditions. In general, PT under microgravity conditions allows subjects to create an increased functional reserve and reduces the effect of unloading observed under real microgravity conditions.

\section{Acknowledgements}

The author wishes to express his appreciation to all who contributed to the success of the experiment. He is especially grateful to V. Stepanstov for his guidance as the IBMP representative of the Exercise Countermeasures Project and the exercise testing group, including I. Amelin, N. Kharitonov, and N. Serikov.

The author gratefully acknowledges the eight women subjects that endured the 120-day confinement.

This work was supported by the Fonds Institute of Biomedical Problems. 


\section{References}

Akima H., Ushiyama J., Kubo J., Tonosaki S., Itoh M., Kawakam Y., Fukuoka H., Kanehisa H., Fukunaga T. Resistance training during unweighting maintains muscle size and function in human calf. Med. Sci. Sports Exerc. 2003; 35: 655-662.

Almeida-Silveira M.I., Pérot C., Pousson M. \& Goubel F. Effects of stretch-shortening cycle training on mechanical properties and fibre type transition in the rat soleus muscle. Pflug. Arch. 1994; 427: 289-294.

Alway S.E., MacDougall J.D., Sale D.G., Sutton J.R., McComas A.J. Functional and structural adaptations in skeletal muscle of trained athletes. J. Appl. Physiol. 1998; 64: 1114-1120.

Buchanan C.I., Marsh R.L. Effects of long-term exercise on the biomechanical properties of the Achilles tendon of guinea fowl. J. Appl. Physiol. 2001; 90: 164-171.

Bojsen-Møller J., Magnusson S.P., Rasmussen L.R., Kjaer M., Aagaard P. Muscle performance during maximal isometric and dynamic contractions is influenced by the stiffness of the tendinous structures. J. Appl. Physiol. 2005; 99: 986-994.

Cavanagh P.R., Komi P.V. Electromechanical delay in human skeletal muscle under concentric and eccentric contractions. Eur. J. Appl. Physiol. 1979; 42: 159-163.

Cé E., Rampichini S., Agnello L., Limonta E., Veicsteinas A., Esposito F. Effects of temperature and fatigue on the electromechanical delay components. Muscle Nerve. 2013; 47: 566-576.

Costa P.B., Ryan E.D., Herda T.J., Walter A.A., Hoge K.M., Cramer J.T. Acute effects of passive stretching on the electromechanical delay and evoked twitch properties. Eur. J. Appl. Physiol. 2010; 108: 301-310.

Ducomps C., Mauriege P., Darche B. Combes S., Lebas F., Doutreloux J.P. Effects of jump training on passive mechanical stress and stiffness in rabbit skeletal muscle: role of collagen. Acta Physiol. Scand. 2003; 178: 215-224.

Eremin A.I., Bazhanov V.V, Marishchuk V.L., Stepantsov V.I., Dzhamgarov T.T. Physical training man in the long-term hypokinesia. In: Probl Kosm Biol, ed. O. Gazenko. M. Nauka. 1969; 191-199.

Esposito F, Limonta E, Cè E. Passive stretching effects on electromechanical delay and time course of recovery in human skeletal muscle: new insights from an electromyographic and mechanomyographic combined approach. Eur. J. Appl. Physiol. 2011; 111: 485-495.

Fernandez-Gonzalo R., Irimia J.M., Cusso R., Gustafsson T., Linné A., Tesch P.A. Flywheel resistance exercise to maintain muscle oxidative potential during unloading. Aviat. Space Environ. Med. 2014; 85: 694-699.

Finni T., Komi P.V., Lukkariniemi J. Achilles tendon loading during walking: application of a novel optic fiber technique. J. Appl. Physiol. 1998; 77: 289-291.

Fitts R.H., Riley D.R., Widrick J.J. Physiology of a microgravity environment invited review: microgravity and skeletal muscle. J. Appl. Physiol. 2000; 89: 823-839.

Fukunaga T., Kubo K., Kawakami Y., Fukashiro S., Kanehisa H., Maganaris C.N. In vivo behavior of human muscle tendon during walking. Proc. R. Soc. Lond. B Biol. Sci. 2001); 268: 229-233.

Goubel F., Marini J.F. Fibre type transition and stiffness modification of soleus muscle of trained rats. Pflug. Arch. 1987; 410: 321-325.

Gopalakrishnan R., Genc K.O., Rice A.J., Lee S.M.C., Enans H.J., Maender C.C., Ilaslan H., Cavanagh P.R. Muscle volume, strength, endurance, and exercise loads during 6-month missions in space. Aviat. Space Environ. Med. 2010; 81: 91-102.

Granata K.P., Ikeda A.J., Abel M.F. Electromechanical delay and reflex response in spastic cerebral palsy. Arch. Phys. Med. Rehabil. 2000; 81: 888-894.

Grigor'yeva L.S., Kozlovskaya I.B. Effects of weightlessness and hypokinesia on velocity and strength properties of human muscles. Kosm. Biol. Aviakosm. Med. 1987; 21: 27-30.

Grosset J.-F., Julien P., Lambertz D., Pérot Ch. Paired changes in electromechanical delay and musculo-tendinous stiffness after endurance or plyometric training. Eur. J. Appl. Physiol. 2009; 105: 131-139.

Grosset J.-F., Thomas L., Mora I., Verhaeghe M., Doutrellot P.-L., Pérot Ch. Follow-up of ankle stiffness and electromechanical delay in immobilized children: Three cases studies. J. of Electromyography and Kinesiol. 2010; 20: 642-647.

Hansen P., Aagaard P., Kjaer M., Larsson B., Magnusson S.P. Effect of habitual running on human Achilles tendon load-deformation properties and cross-sectional area. J. Appl. Physiol. 2003; 95: 2375-2380.

Hill A.V. The heat of shortening and the dynamic constants of muscle. Proc. Royal. Soc. Lond. Ser B Containing Papers of a Biol. Character. 1938; 126: 136-195.

Hof A.L., and van den Berg J.W. EMG to force processing I: an electrical analog of the Hill muscle model. J. Biomech. 1981; $14: 747-58$. 
Hopkins J.T., Feland J.B., Hunter I. A comparison of voluntary and involuntary measures of electromechanical delay. Int. J. Neurosci. 2007; 117: 597-604.

Horita T., Ishiko T. Relationships between muscle lactate accumulation and surface EMG activities during isokinetic contractions in man. Eur. J. Appl. Physiol. 1987; 56: 18-23.

Houston M.E., Norman R.W., Froese E.A. Mechanical measures during maximal velocity knee extension exercise and their relation to fibre composition of the human vartus lateralis muscle. Eur. J. Appl. Physiol. 1988; 58: 1-7.

Koryak Yu.A. The research of velocity-strength properties of human muscular apparatus. Reserved Possibility of Sportsmen Organism. (Karazhanov B ed) Acad Press. Alma-Ata. 1985; 86-87.

Koryak Yu.A. Functional properties of the neuromuscular system in sportsmen of various specializations. Human Physiology 1993; 19: 95-104.

Koryak Yu. Contractile properties of the human triceps surae muscle during simulated weightlessness. Eur. J. Appl. Physiol. 1995; 70: 344-350.

Koryak Yu.A. Effect of 120 days of Bed rest with and without countermeasures on the mechanical properties of the triceps surae muscle in young women. Eur. J. Appl. Physiol. 1998; 78: 128-135.

Koryak Yu.A. Neuromuscular changes under the effect of 7 day mechanical unloading of the human muscular system. Fundamental Studies. 2008; No. 9: 8-21.

Koryak Yu. The adaptation of human skeletal muscles to load changes. Experimental study. Lap Lambert Academic Publishing GmbH \& Co, KG, Germany, 2011a; 402 P.

Koryak Yu Neuromuscular adaptation to short-term and long space flights of the man. In: International Space Station. Russia Segment, eds. A. Grigorev, I. Ushakov. M. SSC-IMBP RAS. 2011b; 2: 93-123.

Koryak Yu.A. Contractile properties and musculo-tendinous stiffness of the triceps surae muscle and their changes as a result of continuous bed rest. Physiol. J. 2012; 58: 66-79.

Koryak Yu.A. Influence simulated microgravity on mechanical properties in the human triceps surae muscle in vivo. I. Effect of 120-days of Bed rest without physical training on human muscle musculo-tendinous stiffness and contractile properties in young women. Eur. J. Appl. Physiol. 2014; 114: 1025-1036.

Koryak Yu., Gidzenko Yu., Shuttlerworth M., Zaletin S., Lonchakov Yu., and Shargin Yu. Functional properties of the neuromuscular system and their changes after a 7-day spaceflight in the International Space Station. Achievements in Modern Natural Sci. 2007; No. 12: 149-150.

Kovanen V., Suominen H., Heikkinen E. Connective tissue of "fast" and "slow" skeletal muscle in rats - effects of endurance training. Acta Physiol. Scand. 1980; 108: 173-180.

Kozlovskaya I.B, Grigoriev A.I. Russian system of countermeasures on board of the International Space Station (ISS): the first results. Acta Astronaut. 2004; 55: 233-237.

Kozlovskaya I.B., Grigoryeva L.S., Gevlich G.I. A comparative analysis of the effect of zero gravity and its model on the force-velocity properties and tonus of human skeletal muscles. Space Biol. and Aviaspace Med. 1984; 18: 22-26.

Kubo K., Kanehisa H., Kawakami Y., Fukunaga T. Elastic properties of muscle-tendon complex in long-distance runners. Eur. J. Appl. Physiol. 2000; 81: 181-187.

Kubo K., Kanehisa H., Ito M., Fukunaga T. Effects of isometric training on the elasticity of human tendon structures in vivo. J. Appl. Physiol. 2001; 91: 26-32.

Lacourpaille L., Hug F., Nordez A. Influence of passive muscle tension on electromechanical delay in humans. PLoS One 2013; 8: e53159.

Lambertz D., Perot C., Kaspranski R., Goubel F. Effects of long-term spaceflight on mechanical properties of muscles in humans. J. Appl. Physiol. 2001; 90: 179-188.

Lambertz D., Mora I., Grosset J.F., Pérot C. Evaluation of musculotendinous stiffness in prepubertal children and adults, taking into account muscle activity. J. Appl. Physiol. 2003; 95: 64-72.

LeBlanc A., Lin C., Shackelford L., Sinitsyn V., Evans H., Belichenko O., Schenkman B., Kozlovskaya I., Oganov V., Bakulin A., Hedrick T., Feeback D. Muscle volume, MRI relaxation times (T2), and body composition after spaceflight. J. Appl. Physiol. 2000; 89: 2158-2164.

Less M., Krewer S.E., Eickelberg W.W. Exercise effect on strength and range of motion of hand intrinsic muscles and joints. Arch. Phys. Med. Rehabil. 1977; 58: 370-374. 
MacDougall J.D., Elder G.C.B., Sale D.G., Moroz J.R., Sutton J.R. Effect of strength training and immobilization on human muscle fibres. Eur. J. Appl. Physiol. 1980; 43: 25-34.

Maganaris C.N. Tensile properties of in vivo human tendinous tissue. J. Biomech. 2002; 35: 1019-1027.

Maganaris C.N., Paul J.P. Tensile properties of the in vivo human gastrocnemius tendon. J. Biomech. 2002; 35: $1639-1646$.

Magnusson S.P., Aagaard P., Dyhre-Poulsen P., Kjaer M. Load-displacement properties of the human triceps surae aponeurosis in vivo. J. Physiol. 2001; 531: 277-288.

Malisoux L., Francaux M., Nielens H., Theisen D. Stretch-shortening cycle exercises: an effective training paradigm to enhance power output of human single muscle fibers. J. Appl. Physiol. 2006; 100: 771-779.

Milner-Brown H.S., Stein R.B., Lee R.G. Synchronization of human motor units: possible roles of exercise and supraspinal reflexes. Electroencephalogr. Clin. Neurophysiol. 1975; 38: 245-254.

Muraoka T., Muramatsu T., Fukunaga T., Kanehisa H. Influence of tendon slack on electromechanical delay in the human medial gastrocnemius in vivo. J. Appl. Physiol. 2004; 96: 540-544.

Narici M., Kayser B., Barattini P., Cerretelli P. Effects of 17-day spaceflight on electrically evoked torque and cross-sectional area of the human triceps surae. Eur. J. Appl. Physiol. 2003; 90: 275-282.

Nicogossian A.E. Countermeasures to space deconditioning. Space physiology and medicine, eds. A.E. Nicogossian, C. Huntoon, S.L. Pool. Lea and Febiger. Philadelphia. 1982; 294-311.

Nordez A., Gallot T., Catheline S., Guevel A., Cornu C., Hug F. Electromechanical delay revisited using very high frame rate ultrasound. J. Appl. Physiol. 2009; 106: 1970-1975.

Norman R.W., Komi P.V. Electromechanical delay in skeletal muscle under normal movement conditions. Acta Physiol. Scand. 1979; 106: 241-248.

Norrbrand L., Fluckey J.D., Pozzo M., Tesch P.A. Resistance training using eccentric overload induces early adaptations in skeletal muscle size. Eur. J. Appl. Physiol. 2008; 102: 271-281.

Orizio C., Baratta R.V., Zhou B.H., Solomonow M., Veicsteinas A. Force and surface mechanomyogram relationship in cat gastrocnemius. J. Electromyogr. Kinesiol. 1999; 9: 131-140.

Orizio C., Gobbo M., Veicsteinas A., Baratta R.V., Zhou B.H., Solomonow M. Transients of the force and surface mechanomyogram during cat gastrocnemius tetanic stimulation. Eur. J. Appl. Physiol. 2003; 88: 601-606.

Pousson M., Pérot C., Goubel F. Stiffness changes and fibre type transitions in rat soleus muscle produced by jumping training. Pflüg. Arch. 1991; 419: 127-130.

Reeves N.D., Maganaris C.N., Narici M.V. Effect of strength training on human patella tendon mechanical properties of older individuals. J. Physiol. 2003; 548: 971-981.

Reeves N.D., Narici M.V., Maganaris C.N. In vivo human muscle structure and function: adaptations to resistance training in old age. Exp. Physiol. 2004; 89: 675-689.

Sandler H., Vernikos J. Inactivity: physiological effects. Acad Press, Orlando, Fla. 1986; 1-9.

Sica R.E.P., McComas A.J. An electrophysiological investigation of limb-girdlee and facioscapulohumeral dystrophy. J. Neurol. Neurosurg. Psychiat. 1971; 34: 469-474.

Stepantsov V.I., Tikhonov M.A., Eremin A.V. Physical training as a method of preventing the hypodynamic syndrome. Kosm. Biol. Aviat. Med. 1972; 6: 64-69.

Trappe T.A., Burd N.A., Louis E.S., Lee G.A., Trappe S.W. Influence of concurrent exercise or nutrition countermeasures on thigh and calf muscle size and function during 60 days of bed rest in women. Acta Physiol. 2007; 191: 147-159

Trappe S., Creer A., Minchev K., Slivka D., Louis E., Luden N., Trappe T. Human soleus single muscle fiber function with exercise or nutrition countermeasures during 60 days of bed rest. Am. J. Physiol. 2008; 294: R939-R947.

Trappe S., Costill D., Gallagher P., Creer A,, Peters J.R., Evans H., Relley D.A., Fitts R.H. Exercise in space: human skeletal muscle after 6 months aboard the International Space Station. J. Appl. Physiol. 2009; 106: 1159-1168.

Tesch P.A..Training for bodybuilding. The encyclopedia of sports medicine. Strength and power in sports, ed. P.A. Komi. Blackwell Oxford. 1991; 370-380.

Viitasalo J.T., Komi P.V. Interrelationships between electromyographic, mechanical, muscle structure and reflex time measurements in man. Acta Physiol. Scand. 1981; 111: 97-103.

Vos E.G., Mullender M.G., van Ingen Schenau G.J. Electromechanical delay in the vastus lateralis during dynamic isometric contractions. Eur. J. Appl. Physiol. 1990; 60: 467-471. 
Vos E.G., Harlaar J., van Ingen Schenau G.J. Electromechanical delay during knee extensor contractions. Med. Sci. Sports Exerc. 1991; 23: 1187-1193.

Watt P.W., Kelly F.J., Goldspink D.F., Goldspink G. Exercise induced morphological and biochemical changes in skeletal muscles of the rat. J. Appl. Physiol. 1982; 53: 1144-1151.

Weiss A.D. The locus of reaction time change with set, motivation and age. J. Gerontol. 1965; 20: 60-64.

Winter E.M., Brookes F.B. Electromechanical response times and muscle elasticity in men and women. Eur. J. Appl. Physiol. 1991; 63: 124-128.

Woo S.L., Ritter M.A., Amiel D., Sanders T.M., Gomez M.A., Kuei S.C., Garfin S.R., Akeson W.H. The biomechanical and biochemical properties of swine tendons-long term effects of exercise on the digital extensors. Connect Tissue Res. 1980; 7: 177-183.

Woo S.L., Gomez M.A., Woo Y.K., Akeson W.H. Mechanical properties of tendons and ligaments. II. The relationships of immobilization and exercise on tissue remodeling. Biorheology 1982; 19: 397-408.

Yavuz S.U., Sendemir-Urkmez A., Turker K.S. Effect of gender, age, fatigue and contraction level on electromechanical delay. Clin. Neurophysiol. 2010; 121: 1700-1706.

Zajac F.E. Muscle and tendon: properties, models, scaling, and application to biomechanics and motor control. Crit. Rev. Biomed. Eng. 1989; 17: 359-411.

Zhou S., Lawson D.L., Morrison W.E., Fairweather I. Electromechanical delay in isometric muscle contractions evoked by voluntary, reflex and electrical stimulation. Eur. J. Appl. Physiol. 1995; 70: 138-145.

Cite thils article aS: Koryak Y.A. Influence of Simulated Microgravity on Mechanical Properties in the Human Triceps Surae Muscle in vivo. Central European Journal of Sport Sciences and Medicine. 2015; 11 (3): 125-143. 
